\title{
A gas proportional scintillator counter for thermal neutrons instrumentation
}

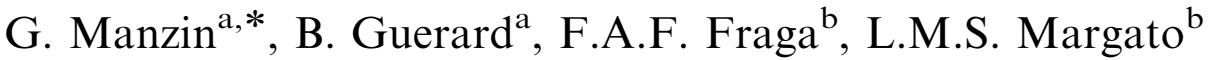 \\ ${ }^{a}$ Institut Laue Langevin, 6 rue Jules Horowitz, 38042 Grenoble, France \\ ${ }^{\mathrm{b}}$ LIP-Coimbra and Departamento de Fisica de la Universidade de Coimbra, 3004-516 Coimbra, Portugal
}

Available online 13 August 2004

\begin{abstract}
A gaseous scintillation detector consisting of a ${ }^{3} \mathrm{He}+\mathrm{CF}_{4}$ filled MSGC detector coupled with an optical readout has been investigated for an application for high-resolution and high counting rate position-sensitive neutron detector. Measurements of the photons' emission as a function of the gas mixture have been carried out indicating that filling the detector with ${ }^{3} \mathrm{He}+\mathrm{CF}_{4}$ is a very good choice also from the point of view of optical readout and not only for charge readout. The light yield has been systematically measured as a function of the $\mathrm{CF}_{4}$ concentration and operating voltages. We also studied the emission of primary light, rather strong in $\mathrm{CF}_{4}$ mixtures, which allows the observation of the light signal even in the absence of high voltage on the electrodes. Finally, some measurements of the position resolution will be presented.
\end{abstract}

(C) 2004 Elsevier B.V. All rights reserved.

PACS: 29.40.Gx; 29.40.Mc

Keywords: GSPC; Optical readout; CCD

\section{Introduction}

It has been demonstrated [1,2] that a MSGC plate, installed in a gas volume suitable for neutron detection, can generate a number of photons during the avalanche process, sufficient for detection via a CCD camera. This is a

\footnotetext{
*Corresponding author.

E-mail address: gmanzin@ill.fr (G. Manzin).
}

consequence of the fact that every avalanche multiplication or charge migration in a gas volume is accompanied by the emission of photons. Those photons are the result of inelastic collisions between charged particles and gas molecules. The use of this light producing phenomenon, never used before in neutron detection, is at the origin of the study of a GSPC detector currently testing carried out at the Institut Laue Langevin in Grenoble. 


\section{Experimental set-up}

The detector used for the measurements described in this paper was made of an MSGC plate assembled in a gas tight stainless steel box. This gas vessel has a diameter of $20 \mathrm{~cm}$ and two opposite windows: an aluminium one, $5 \mathrm{~mm}$ thick, in order to allow the neutrons to enter the gas volume, and a quartz (Suprasil) one, again $5 \mathrm{~mm}$ thick, to allow photons' readout. A special light tight flange is used to position a 1-in. photomultiplier in front of the quartz window. Similarly, it is possible to position a CCD camera for imaging purposes. A schematic drawing of the gas vessel and a more detailed description can be found in Ref. [2]. Several feed-through allow the application of the voltages to the electrodes and the readout of the charge signals. The bidimensional MSGC used for the tests is of the Bidim-bis type developed at the ILL and described in Ref. [3]. For this application the MSGC plate needs to be assembled in the gas vessel in a reverse way compared to a normal neutron detector (i.e., with the cathodes facing the aluminium entrance window) because we need the anodes, where the light is produced, to face the quartz window. All the measurements presented in this paper have been performed on a test beam line, having a total flux of $2.4 \times 10^{7} \mathrm{n} / \mathrm{s}$ at a wavelength of $2.5 \AA$, irradiating the detector through a cadmium slit having different dimensions in the various measurements.

\section{Measurements with ${ }^{3} \mathrm{He}-\mathrm{CF}_{4}$ mixtures}

The measurements with the ${ }^{3} \mathrm{He}-\mathrm{CF}_{4}$ gas mixture had the aim to better understand the light emission process, its dependence on the concentration of the two gases, and the amount of visible and UV light emitted in the process. The first measurement with this gas mixture has been about the variation of the emitted light as a function of the quantity of $\mathrm{CF}_{4}$ present in the gas mixture (see Fig. 1). The amount of ${ }^{3} \mathrm{He}$ was of $100 \mathrm{mbar}$. The light spectra have been recorded with a Hamamatsu R292 UV sensitive photomultiplier to which a high voltage of $750 \mathrm{~V}$ was applied. The MSGC

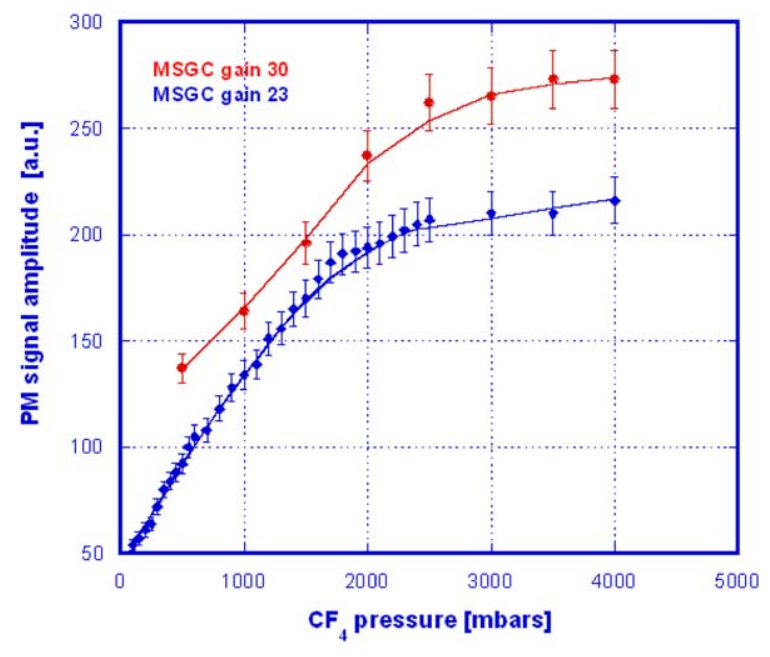

Fig. 1. Signal amplitude of the light signal from the MSGC plate as a function of the $\mathrm{CF}_{4}$ pressure, for $100 \mathrm{mbar}$ of ${ }^{3} \mathrm{He}$ and two different MSGC gains.

gain has been kept constant through the entire measurement by increasing the applied high voltage with the increase of the gas pressure; in other words, keeping the signal amplitude constant. The number of emitted photons reaches a plateau for a $\mathrm{CF}_{4}$ pressure higher than $2.5 \mathrm{bar}$; in order to maximize the light emission, this amount of $\mathrm{CF}_{4}$ would be sufficient but in order to decrease the proton and triton track length it is better to use a higher pressure. The next measurement has been to check the light emission as a function of the $\mathrm{CF}_{4}$ pressure for different ${ }^{3} \mathrm{He}$ concentrations in the gas mixture, in order to see if the ${ }^{3} \mathrm{He}$ has any influence on the number of emitted photons. The measurement conditions were the same as in the previous case. Fig. 2 shows the amplitude of the light signal (recorded with the UV-sensitive photomultiplier) for the different gas mixtures. It is clear that at least at low ${ }^{3} \mathrm{He}$ concentrations the amount of this gas present in the mixture does not affect the light emission.

\subsection{Others gas mixtures}

In the literature it has been often reported [4] that the best scintillating gas mixtures contain big concentrations of pure noble gases, especially xenon and neon. We then wanted to verify if the 


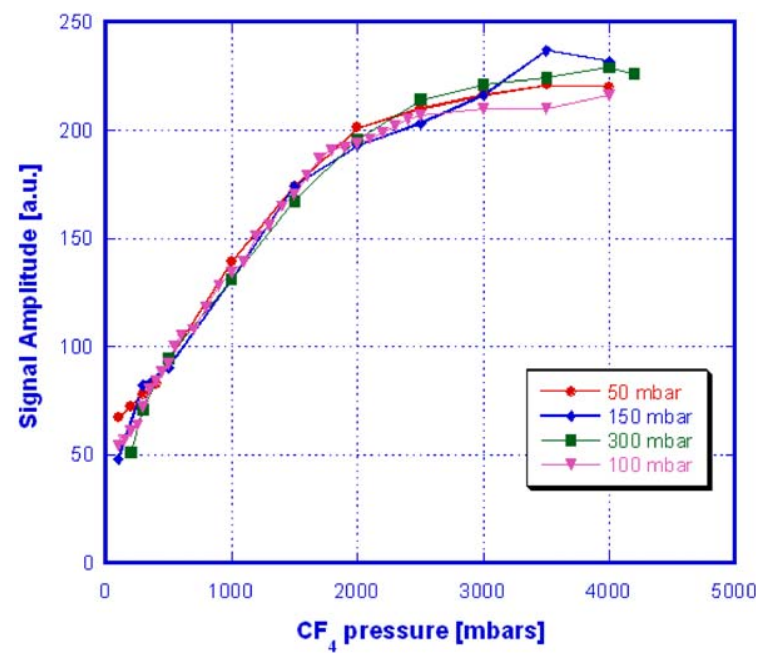

Fig. 2. Signal amplitude of the light signal from the MSGC plate as a function of the $\mathrm{CF}_{4}$ pressure, for $50,100,150$ and 300 mbar of ${ }^{3} \mathrm{He}$.

addition of small amounts of a noble gas to the gas mixture would improve the light emission. The measurement has been performed by starting with a gas mixture of 100 mbar of ${ }^{3} \mathrm{He}$ and 2 bar $\mathrm{CF}_{4}$ to which small amounts of noble gases have been added in order to see if this would increase the amount of emitted light. All the gases used in those tests are of high-grade purity $(99.999 \%)$ and from different producers. Fig. 3 shows the amount of light emitted if an increasing amount of $\mathrm{Ne}$ is added to the standard gas mixture. From this plot it is clear that even a very small amount of neon in the gas mixture has a rather dramatic effect on the amount of light emitted, with decreases of more than $40 \%$ when the amount of neon present in the gas mixture increases from 0 to 400 mbar. A similar effect can be observed if small quantities of xenon are introduced in the standard gas while the addition of small amount of argon does not seem to affect very much the total light emissions, but we observed increased gamma sensitivity of the detector.

\section{Comparison with the GS20 Lithium glass scintillator}

A comparison with the GS20 Lithium glass scintillator [5] has been carried out, in order to

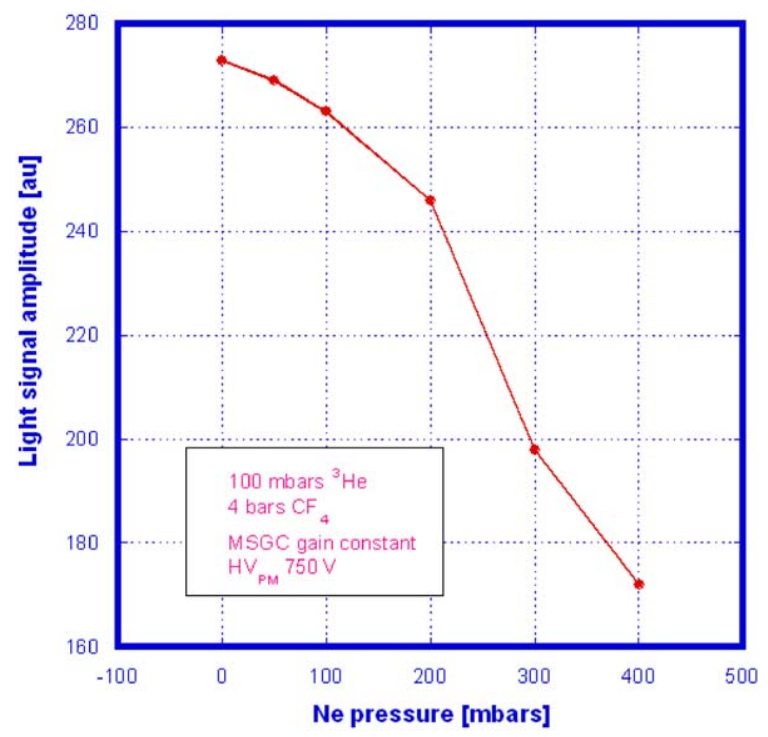

Fig. 3. Signal amplitude of the light signal from the MSGC plate as a function of the $\mathrm{Ne}$ pressure.

establish which one of the two detectors offers the best performances. The same photomultiplier R292 has been used for both measurements, once connected to the MSGC test box via the light tight flange, and then to the GS20 scintillator simply using optical grease. The GS20 scintillator had a diameter of 1 inch and $1 \mathrm{~mm}$ thickness. Both detectors have been irradiated with a neutron beam by using a Cadmium slit of $1 \times 10 \mathrm{~mm}^{2}$ and the gain on the photomultiplier has been kept constant; the MSGC high voltage was of $1900 \mathrm{~V}$. Fig. 4 shows the comparison of the pulse height spectra measured when irradiating the GS20 and the MSGC with the $\mathrm{n}$ beam, with a PM high voltage of $650 \mathrm{~V}$ in both cases, correcting the difference in count rate due to the difference of efficiency of the detectors; for the GS20 it was of $78 \%$ while with only 100 mbar of ${ }^{3} \mathrm{He}$ it was of $6.8 \%$ for the MSGC. We can observe that the performances of the GSPC are better than the GS20 scintillator ones. While energy resolution and counting rates are comparable, the gamma discrimination, a known weak point for neutron scintillators, is much better for the GSPC. 


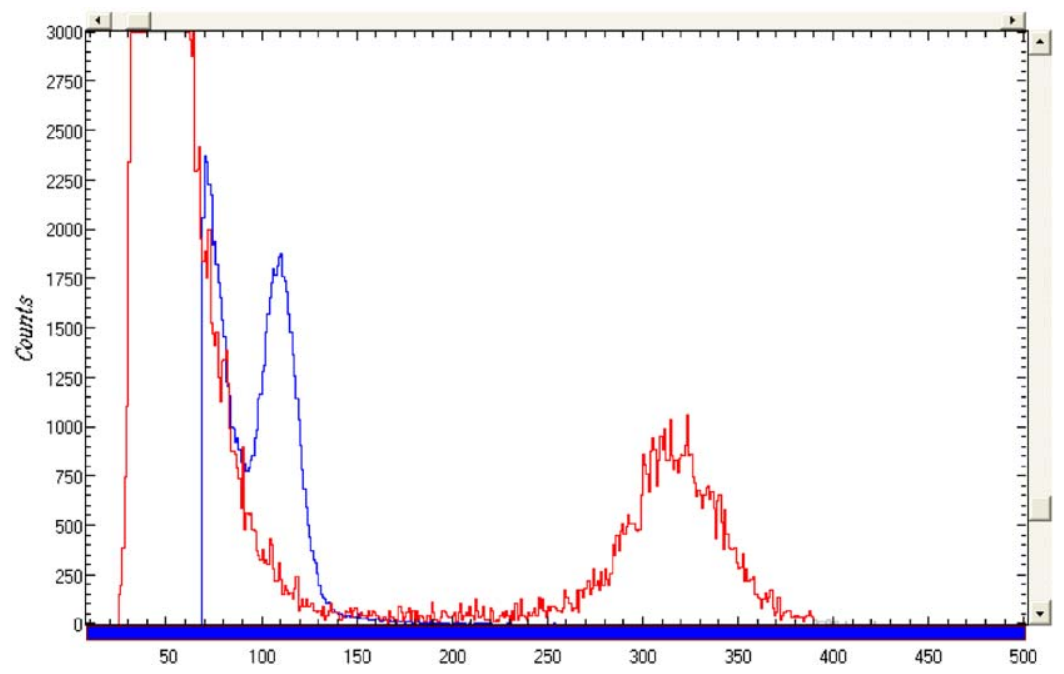

Fig. 4. Comparison between the GS20 and MSGC light spectra obtained irradiating the detector with a thermal neutron beam; the MSGC spectrum has been corrected in order to simulate a $78 \%$ efficiency.

\section{CCD measurements}

The detector was then coupled to a high sensitivity CCD camera via a $50 \mathrm{~mm}$ lens, f 1.8 , positioned at $33 \mathrm{~cm}$ from the MSGC plate, in order to focus on the anodes where the maximum light should be emitted. All the measurements have been realized on a test beam line with a Cadmium slit of $1 \times 2 \mathrm{~cm}^{2}$. With a gas mixture of $100 \mathrm{mbar}$ of ${ }^{3} \mathrm{He}$ and 1 bar $\mathrm{CF}_{4}$ we have been able to measure the intensity of the light emitted in the gas volume as a function of the MSGC high voltage and thanks to the possibility of a simultaneous readout of the charge and light signals, compare it to the MSGC gain. From Fig. 5 we can note that it is possible to observe photons emitted in the gas volume well before avalanche multiplication takes place in the detector; in fact even when no high voltage is applied to the MSGC. This means that the interaction of the charged particles generated by the neutron absorption in the gas volume is enough to produce photons and their number is sufficient for the signal to be seen by the CCD camera in a relatively short acquisition time of $20 \mathrm{~s}$. The same effect can be observed in the recorded CCD images; Fig. 6 shows the slit image

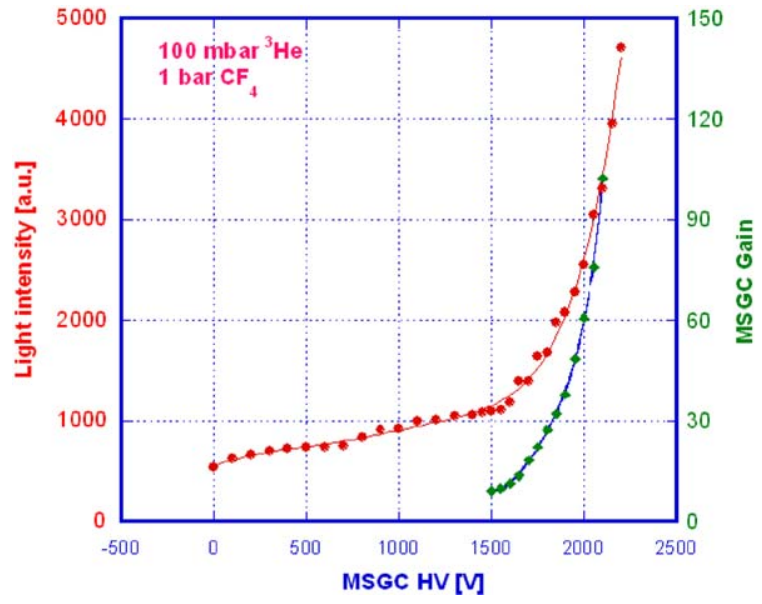

Fig. 5. Light intensity and MASGC charge gain as a function of the MSGC high voltage.

obtained with no high voltage applied to the MSGC and the horizontal projection of the 2D image. The edges of the image are rather well defined and the light emission uniform across the slit. Applying a high voltage of $2700 \mathrm{~V}$ to the MSGC, we can observe from Fig. 7 that the 


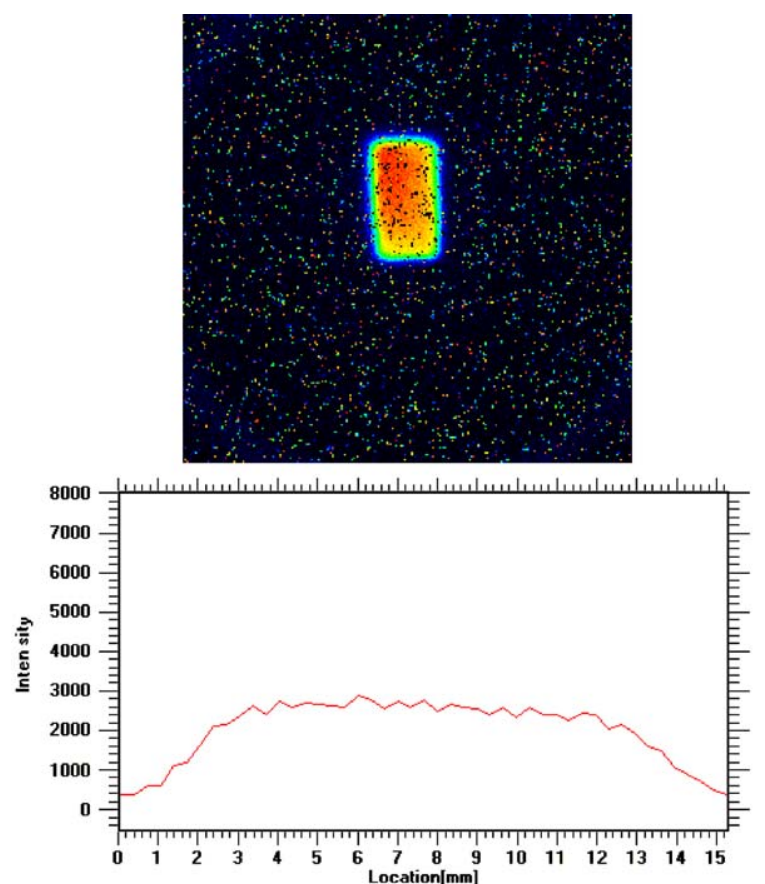

Fig. 6. Image of the light emitted by the GSPC irradiated through a $1 \times 2 \mathrm{~cm}^{2}$ Cadmium slit when no high voltage is applied to the MSGC electrodes and the horizontal projection of the image.

number of collected photons is of course much higher for the same acquisition time and also that the anode structure is now easily visible. This is explained by the fact that the avalanche electron multiplication takes place in the immediate vicinity of the anodes strips so this is also the location of the maximum light emission. The image with the high voltage on the MSGC plate clearly shows that the system is easily able to resolve light sources inside the detector as close as $1 \mathrm{~mm}$. This suggests that by using a structure with a finer pitch it would be possible to obtain a better position resolution.

\section{Conclusions}

Some systematic measurements of the light emission in different gas mixtures used in neutron detection show that ${ }^{3} \mathrm{He}-\mathrm{CF}_{4}$ is an excellent gas

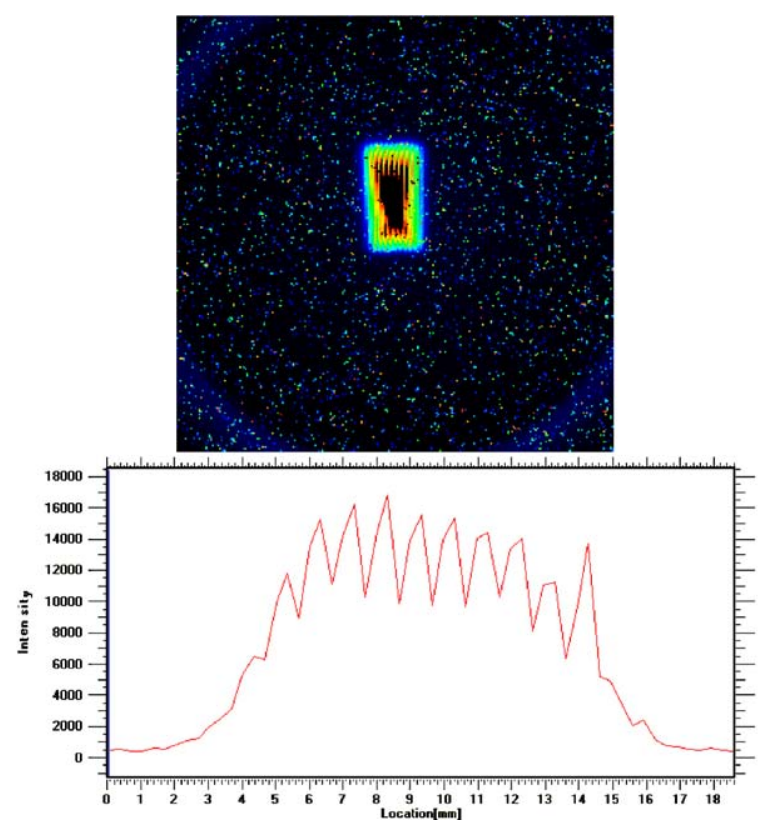

Fig. 7. Image of the light emitted by the GSPC irradiated through a $1 \times 2 \mathrm{~cm}^{2}$ Cadmium slit with a high voltage of $2700 \mathrm{~V}$ applied to the MSGC electrodes and the horizontal projection of the image.

mixture also from the point of view of the scintillation and not only for charge readout. The addition of other gases to this mixture did not enhance the light emission. An interesting feature of this device is the possibility of observing the primary light emission, i.e., the light emitted during the interaction of the proton and the triton (generated by neutron absorption in the gas) with the $\mathrm{CF}_{4}$, without application of any high voltage to the electrodes. Finally, a preliminary position resolution of $1 \mathrm{~mm}$ corresponding to the strips pitch has been measured.

\section{References}

[1] F.A.F. Fraga, L.M.S. Margato, S.T.G.G. Fetal, M.M.F.R. Fraga, R. ferreira Marques, A.J.P.L. Policarpo, B. Guerard, A. Oed, G. Manzin, T. van Vuure, Nucl. Instr. and Meth. A 475 (2002) 357. 
[2] G. Manzin, Development of a gas scintillator system for neutrons detection, $\mathrm{PhD}$ Thesis, Politecnico di Milano (2004).

[3] N. Vellettaz, J.E. Assaf, A. Oed, Nucl. Instr. and Meth. A 392 (1997) 73.

[4] V. Peskov, G. Charpak, W. Dominik, F. Sauli, Nucl. Instr. and Meth. A 277 (1989) 547.
[5] J-F. Clergeau, B. Guerard, J.C. Buffet, P. Van Esch, High position resolution neutron detector using a position sensitive photomultiplier tube, in: I. Anderson, B. Guerard (Eds.); Advances in Neutron Scattering Instrumentation, Proc. SPIE 4785 (2002) 254. 